\title{
Perspectivas da educação física para a população idosa
}

\section{Perspectives of physical education for the elderly population}

\section{Perspectivas de la educación física para la población anciana}

\author{
Clarissa Biehl-Printes $\left(\mathbb{1}\right.$ ', Fabiane de Oliveira Brauner $(1) \square$, Newton Luiz Terra ${ }^{1}{ }^{1}$ \\ ' Pontifícia Universidade Católica do Rio Grande do Sul (PUCRS). Porto Alegre, RS, Brasil.
}

\section{ARTICLE INFO}

\section{Article history}

Received: 21/11/2018

Accepted: 02/05/2019

Published: 29/08/2019

\section{$\triangle$ Correspondent Author}

Fabiane de Oliveira Brauner

Instituto de Geriatria e Gerontologia/PUCRS

Av. Ipiranga, 6690, prédio 81, sala 703

90610-000, Porto Alegre, RS, Brasil

braunerpro@gmail.com

\section{(C) 2019 All rights reserved}

\section{Editors}

Alfredo Cataldo Neto

Newton Luiz Terra

Assistant Editors

Paula Engroff

Vanessa Sgnaolin

\begin{abstract}
RESUMO
OBJETIVOS: Caracterizar: i) o papel da educação física na população idosa, ii) os programas e as propostas de exercício físico que são utilizados no Brasil nos últimos 10 anos e iii) as perspectivas da educação física na população idosa.

DELINEAMENTO: Revisão de literatura e resumos de Anais (2007-2017) conduzidos nas bases PubMed, MEDLINE, SciELO e Scholar-Google por meio dos seguintes descritores: exercício físico, educação física, expectativa de vida, anos de vida saudável, entre outros. Livros com teor científico também foram consultados. Após, realizou-se uma análise crítica sobre os estudos encontrados na forma de texto descritivo.

CONCLUSÃO: A educação física tem reconhecida pertinência no envelhecimento, sendo que novas perspectivas se estabelecem no empoderamento político e social em promoção da saúde no idoso. Os programas de atividade física carecem alargar oportunidades e evidenciar resultados de efetividade/eficácia.
\end{abstract}

DESCRITORES: Educação física; Idosos; Exercício físico; Atividade física; Estilo de vida.

\begin{abstract}
OBJECTIVES: To characterize: i) the role of physical education in the elderly population; ii) the programs and physical exercise proposals that have been used in Brazil in the last 10 years; and iii) the perspectives of physical education in the elderly population.

DESIGN: Literature review and abstracts of Annals since 2007 conducted in PubMed, MEDLINE, SciELO and Scholar-Google databases through the following descriptors: physical exercise, physical education, life expectancy, years of a healthy life, among others. Books with scientific content were also consulted. Afterward, a critical analysis was performed on the studies found in descriptive text.

CONCLUSION: Physical education has recognized relevance in aging, and new perspectives are established in the political and social empowerment in health promotion in the elderly. Physical activity programs need to widen opportunities and evidence of effectiveness/effectiveness results.

KeYwords: Physical education; Elderly; Physical exercise; Physical activity; Life style.
\end{abstract}

\section{RESUMEN}

OBJETIVOS: Caracterizar: i) el papel de la educación física en la población anciana, ii) los programas y las propuestas de ejercicio físico que se utilizan en Brasil en los últimos 10 años y iii) las perspectivas de la educación física en la población anciana.

DISEÑO DEL ESTUDIO: Revisión de literatura y resúmenes de Anais (2007-2017) llevado a cabo en las bases PubMed, MEDLINE, SciELO y Scholar-Google por medio de los siguientes descriptores: el ejercicio físico, la educación física, la expectativa de vida, los años de vida sana, entre otros. Los libros con contenido científico también fueron consultados. Después, se realizó un análisis crítico sobre los estudios encontrados en la forma de texto descriptivo.

CONCLUSIÓN: La educación física tiene reconocida pertinencia en el envejecimiento, siendo que nuevas perspectivas se establecen en el empoderamiento político y social en promoción de la salud en el anciano. Los programas de actividad física carecen de ampliar oportunidades y evidenciar resultados de efectividad/eficacia.

Plabras CLAVE: Educación física; Ancianos; Ejercicio físico; Entrenamiento físico; Estilo de vida. 


\section{INTRODUÇÃO}

A população mundial está envelhecendo num ritmo exponencial, e o Brasil é um dos países com maior crescimento. A Organização Mundial da Saúde (OMS) estima que o Brasil aumente dos atuais 14\% para 20\% da população idosa em menos de 20 anos, enquanto países europeus como a França levaram mais de 100 anos para atingirem esse percentual ${ }^{1}$. A população idosa brasileira aumentou 43\% entre os anos de 2000 e 2010, e a população longeva (com 80 anos ou mais) atingiu um crescimento ainda mais importante $(75,9 \%)$. O país encaminha-se para ter a sexta população mais idosa do mundo até $2025 .^{2}$

A grande quantidade de idosos juntamente com a maior expectativa de vida são os principais desafios da saúde, visto que isso traz mudanças significativas em outros aspectos, como as morbidades e comorbidades, a inclusão social, participação na sociedade, segurança e condições de vida. Trata-se de uma diversidade de fatores que espelham a emergente necessidade de aplicação dos objetivos colocados pela Organização Mudial de Saúde (OMS): aumentar os anos vividos com saúde através de uma prática de envelhecimento ativo.

A atuação do profissional de educação física perante essa mudança populacional torna-se cada vez mais significativa. A diversidade e as potencialidades de programas de atividade física regular oferecidos a nível nacional e destinados à dita Terceira Idade atendem ao que se pretende em envelhecimento ativo de acordo com a OMS: otimização das oportunidades para a saúde, participação e segurança, para melhorar a qualidade de vida das pessoas que envelhecem. ${ }^{3,4}$

Diante da variedade de implicações que o aumento da população idosa traz, incluindo desafios também econômicos e sociais, faz-se necessário, numa perspectiva de efetividade do exercício físico, atuar sobre a redução da distância entre as curvas de morbidade e mortalidade. Considerando a expectativa de vida atual do brasileiro, em aproximadamente 76 anos, esse idoso conta em média com mais 4,6 anos de expectativa livre de doenças crônicas e 17,2 anos livre de incapacidade funcional depois dos 60 anos. ${ }^{5}$ Conforme essas evidências, a educação física tem um papel fundamentel no manejo das doenças crônicas, assim como na correção e prevenção de fatores de risco para a saúde, típicos no indivíduo idoso.

Tanto a literatura especializada como a pluralidade de encontros científicos atuais, nacionais e internacionais, que discutem o processo de envelhecer, são incansáveis, unânimes e repetitivos em transmitirem a preponderância do papel do exercício físico no impacto da saúde das pessoas idosas.,6,7 Uma das potencialidades da prática regular do exercício físico é atuar de forma indireta sobre aspectos psicológicos (autoestima, autoeficácia) e nas relações

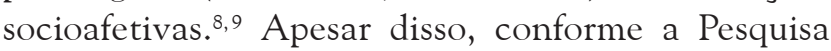
Nacional de Saúde ${ }^{10}$, o idoso brasileiro ( $>60$ anos ou mais) ainda "mexe-se" muito pouco: 78,9\% não pratica exercício físico ou esporte de forma regular. Apenas $23 \%$ atendem ao aconselhamento do médico para praticarem exercício físico. ${ }^{10}$

Por outro lado, numa perspectiva científica, é descrito na literatura que existe uma carência em mostrar evidências de custo-utilidade e custoefetividade no caso de programas públicos, assim como os desenvolvidos no campo acadêmico. ${ }^{3} \mathrm{O}$ papel da educação física na população idosa hoje necessita atuar sinergicamente na educação e saúde, mostrar mais informações, resultados e evidências sobre o impacto na saúde do idoso (impacto sobre o envelhecimento biológico) e na saúde pública (impacto sobre o envelhecimento populacional).

Os programas de estímulo à prática de exercício físico ou esporte são estratégias de ação muito eficazes no combate ao sedentarismo e controle das Doenças Crônicas Não Transmissíveis (DCNT), além da oportunidade de participação e inclusão social. ${ }^{10} \mathrm{As}$ próximas décadas serão promissoras para um papel de maior importância tanto para o exercício físico como para a atuação do profissional de educação física. A necessidade da inserção do profissional de educação física e de programas de exercício físico nas Unidades de Saúde (US) do Instituto Nacional do Seguro Social (INSS) parece assumir uma realidade cada vez mais emergente. ${ }^{11,12}$

Essa realidade da mudança populacional, condutual da pessoa idosa (aderência e permanência no exercício físico) e da qualificação do profissional de educação física traz as seguintes reflexões: estamos preparados para envelhecer? O brasileiro está sabendo envelhecer? O profissional de educação física está preparado para atuar com efetividade sobre a saúde da pessoa idosa?

Conforme o exposto, este estudo tem como principais objetivos caracterizar, por meio de uma revisão da literatura, o papel da educação física na população idosa, as características dos programas de exercício físico que são utilizados no Brasil nos últimos 10 anos e as perspectivas da educação física na população idosa.

O presente trabalho é uma revisão de literatura, que buscou analisar estudos dos últimos anos (20072017). A busca bibliográfica foi conduzida nas bases PubMed, MEDLINE (http://www.ncbi.nlm. nih.gov/ pubmed), SciELO (http:// www.scielo.org) e Scholar- 
Google (http://www.scholar.google.com). Utilizou-se a combinação dos seguintes descritores: exercício físico, atividade física, programas de exercício para idosos, idosos, envelhecimento, educação física, expectativa de vida, anos de vida saudável. Para a análise dessa revisão foram incluídos artigos que correspondessem aos seguintes critérios: 1) artigos originais, resumos de Anais e livros com teor científico; 2) programas brasileiros de exercício físico para pessoas idosas acima de 60 anos; 3) participação e supervisão do profissional de educação física; 4) língua portuguesa e inglesa.

Foram encontrados inicialmente 49 artigos na base de dados PubMed, MEDLINE, 17 artigos na SciELO e 22 artigos e resumos de Anais no ScholarGoogle. Também foram consultados 9 livros com teor científico. Somando 97 estudos no total. Foram excluídos 51 artigos e Anais com mais 3 livros científicos por não se encaixarem nos critérios de busca visto que os programas de exercício não se destinavam exclusivamente para pessoas idosas; não se enquadravam no espaço temporal determinado para os últimos 10 anos; não direcionavam de forma objetiva a participação do profissional de educação física de forma a não se aplicar aos critérios de inclusão do estudo. Por fim, estavam de acordo com os critérios de seleção 37 artigos e Anais com mais 6 livros científicos que foram incluídos na presente revisão. A seguir, foi realizada a análise crítica sobre os estudos encontrados na forma de texto descritivo sobre o referente assunto.

\section{REVISÃO DA LITERATURA}

\section{O papel da educação física na população idosa}

A educação física na velhice está intimamente associada à prática de atividade física regular em benefício da saúde, manutenção da independência, autonomia e desenvolvimento de relações socioafetivas. ${ }^{13}$

O aumento no perfil demográfico tem contribuído para aumentar o número de estudos da relação entre o envelhecimento saudável e a prática de exercício físico. ${ }^{3}$ Na pesquisa científica sobre a prática da educação física e o envelhecimento tem predominado a análise dos efeitos da prática regular de exercício sobre a prevenção de doenças, tais como: hipertensão, dislipidemia, diabetes melito e doenças cardíacas. Esse tem sido também um dos principais ângulos de análise quando se desenvolvem campanhas de saúde pública centradas na atividade física. ${ }^{14}$ Graças aos esforços científicos das últimas décadas, é hoje reconhecido pelas comunidades científica, médica e pela generalidade da sociedade civil o papel relevante da atividade física regular/exercício físico na saúde e no bem-estar das pessoas de todas as idades.

Sendo assim, o exercício físico, como parte integrante do amplo conceito de atividade física, tem sido considerado como o melhor contributo para um envelhecimento saudável. ${ }^{15}$ É importante destacar que não existe outro segmento da população que mais se beneficie da prática de exercício físico regular do que os idosos. Chodzko-Zajko ${ }^{16}$ afirma que o idoso poderá sair em vantagem desse processo natural se for social, intelectual e fisicamente ativo.

Nesse contexto, as práticas de educação física pela população idosa se dão por meio de programas ou propostas de promoção da saúde relacionadas ao exercício físico regular. ${ }^{12}$ Para alguns autores, elas são consideradas estratégias de intervenção que deverão atender a um envelhecimento saudável, por isso precisam ser priorizadas nas intervenções realizadas nas unidades de saúde da família, uma vez que, além dos benefícios físicos, psicológicos e sociais, podem gerar grande economia no setor da saúde. ${ }^{17,18,19}$ A literatura também mostra que esses programas poderão decorrer no formato formal em programas comunitários municipai ${ }^{20}$, ou programas no âmbito de desenvolvimento de projetos de pesquisa acadêmico, ou inseridos em Universidade Aberta para Terceira Idade. ${ }^{21,22}$ Como também, num formato em que o exercício da profissão decorre num caráter comercial em que a prática de exercício físico se dá pela relação do profissional de educação física e o idoso por prestação de serviço no modo individual, coletivo num centro/academia para prática de exercício físico supervisionado. ${ }^{23-30}$

Consoante os formatos, informal ou comercial, o papel da educação física na população idosa deverá ser cercado de conhecimentos em gerontologia preventiva e social para que seja possível atender às suas características e necessidades. Conforme é descrito em diversos estudos prévios, os programas quando constituídos por acolhimento, avaliação da aptidão física, da condição funcional, seguido de orientação, prescrição do exercício e acompanhamento supervisionado dos participantes atingem efeitos sobre a saúde e o bem-estar desses indivíduos. ${ }^{3,31,32,33}$

Compreendendo que existe uma relação muito próxima entre o envelhecimento e o acometimento e desenvolvimento de doenças geriátricas, tornase evidente a necessidade de existir supervisão na prática de exercício físico ao idoso. ${ }^{34}$ Diversos estudos demonstram que a falta de supervisão impacta na baixa adesão e permanência. ${ }^{35}$ Essas propostas não supervisionadas espelham o resultado de um "aconselhamento" por parte do médico assistente para 
prática de uma atividade física ou exercício, que, por sua vez, nem sempre garante a adesão nem o efeito que se busca do exercício na saúde, visto ainda que a indicação para realização de exercício físico regular em algumas doenças será de leve intensidade. ${ }^{36} \mathrm{Em}$ estudo prévio, é colocada a diferença estatística entre o médico aconselhar e recomendar para o engajamento no exercício físico, uma vez que a recomendação com orientação de local, profissional e programa toma uma dimensão superior. ${ }^{35,37}$

Nesse sentido, já que as evidências científicas e indicações para que o idoso pratique exercício físico assumem o protagonismo quando se trata de Geriatria e Gerontologia preventivas, há interesse em que seja dada a devida importância para o exercício físico, assim como para a inserção e o papel do profissional de educação física nessa população. Essa importância deverá ser essencialmente transmitida pelo médico assistente e compreendida pelo idoso de forma a favorecer a mudança de comportamento.

A literatura deixa bem esclarecido que o papel da educação física para a saúde da pessoa idosa cada vez mais assume uma participação preventiva, recuperadora e de prolongamento das funções físicas e cognitivas diminuídas ou afetadas associadas à idade, assim como assume um papel evidente de promoção da saúde e qualidade de vida. ${ }^{3,8,9}$

\section{As características dos programas de exercício físico que são utilizados no Brasil nos últimos 10 anos}

Considerando que a saúde do idoso está amplamente relacionada com hábitos de vida e nível de atividade física e/ou exercício físico, foi possível verificar que os programas direcionados para essa população no Brasil têm maior ocorrência em local acadêmico (universidade/laboratório). Programas realizados na comunidade ainda são pouco documentados. ${ }^{3,10}$ Corroborando com a literatura, importa colocar que os programas quando decorrem no campo acadêmico cumprem com critérios de inclusão e exclusão. Nesse sentido, para além de os participantes serem selecionados e habitualmente representarem grupos com características homogêneas e indivíduos motivados, os programas são conduzidos em ambiente clínico e/ou em condições controladas. ${ }^{3}$ Devido ao rigor e à qualidade dos estudos analisados de natureza quantitativa, com intervenções predominantemente tradicionais, ficou demonstrado, pela grande maioria, a eficácia das atividades desenvolvidas em ambientes controlados ou formais (universidades, etc) sobre o incremento nos aspectos relacionados à aptidão física e outros. $3,32,33,38,39,40$
Destaca-se na literatura um único estudo de revisão que utlizou o modelo de análise RE-AIM praticado no Brasil, destinado a analisar tanto a validade externa quanto a validade interna de programas de saúde. ${ }^{3}$ Esta revisão propôs uma avaliação da produção científica brasileira sobre os programas de atividade física para idosos. Segundo os autores, os programas atingem resultados de total eficácia quando alcançam as cinco dimensões: alcance (número absoluto, proporção do público-alvo atendido e sua representatividade no programa e avaliação das características do públicoalvo); efetividade/eficácia (relatórios dos resultados primários, relatos dos mediadores, consequências negativas, custo-efetividade); adoção (proporção e perfil da equipe, dos praticantes e dos planos adotados na intervenção); implementação (é a fidelidade da intervenção por parte das organizações e dos sujeitos); e manutenção (quanto o programa se estendeu após sua implementação) ${ }^{3}$

Para esses pesquisadores, a limitação de os programas acadêmicos cumprirem com todas as dimensões está nos próprios desenhos dos estudos e das propostas de programas, visto as barreiras para adoção (cumprimento de critérios de inclusão/exclusão), além da manutenção por ter um início e um fim estabelecidos e lacunas em efetividade/eficácia, já que não são demonstrados resultados sobre consequências negativas e custo-efetividade, sendo este último tão útil em saúde pública. ${ }^{3}$

Nesses mesmos achados, ainda salientaram que um programa comunitário publicado foi o que mais atendeu as cinco dimensões do RE-AIM. ${ }^{3,31}$ No entanto uma sistemática de revisão voltada para os benefícios da atividade física e qualidade de vida em idosos de comunidade salientou sobre a necessidade de mais estudos longitudinais para a obtenção de resultados mais robustos a esse respeito. ${ }^{38}$ Para além desse estudo de revisão, um outro estudo encontrado também reforça a necessidade de os programas de intervenção de atividade física contemplarem as cinco dimensões do modelo RE-AIM de avaliação.,39 A necessidade de mais qualidade, ao ser demonstrada nas evidências dos programas de atividade física para idosos, é uma importante lacuna a ser melhorada em futuros trabalhos.

Além da educação física atender as pessoas idosas através de programas acadêmicos e comunitários, conta-se com a prática em ambiente de centro/ academia/clube de esporte/saúde ou ainda através de personal trainer. São propostas que acolhem e acompanham as pessoas idosas e que igualmente são documentadas através de estudos, sendo mais comum encontrar sobre os efeitos da hidroginástica na saúde, 
autoestima; nas modalidades de musculação, ginástica geral/funcional ou ainda estudos de caso voltados ao âmbito acadêmico, este último citado em menor quantidade. ${ }^{24-30}$ No conjunto desses estudos, observou-se que os idosos foram submetidos a procedimentos de acolhimento, avaliação da aptidão física e orientação e prescrição dos exercícios, cercando-se de conhecimentos e procedimentos nas bases da fisiologia do exercício, biometria, metodologia do treino e outros voltados à avaliação da autoestima e qualidade de vida. Com base nessa literatura divulgada, vê-se uma ampla forma de atuação da área da educação física na pessoa idosa, atingindo metas de atuação em envelhecimento ativo. Por outro lado, considerando a relevância da abordagem entre eficácia de exercício físico na pessoa idosa o formato comercial de aplicação de programas deveria ser mais explorado e exposto na literatura.

\section{As perspectivas da educação física na população idosa}

O paradigma "envelhecimento ativo" adotado pela Organização Mundial da Saúde (OMS) no final do século XX, perante os desafios que a maior longevidade acarreta, é entendido como processo de cidadania plena em que se otimizam oportunidades de participação, segurança e uma maior qualidade de vida à medida que as pessoas vão envelhecendo (WHO).${ }^{4}$ Essa definição contemporânea do processo de envelhecer sai de uma visão reativa, centrada nas necessidades básicas em que a pessoa é um agente passivo, para uma proativa, que reconhece a pessoa como um elemento capaz e atuante na sociedade..$^{41}$ Esse protótipo colocado pela OMS expõe um modelo alargado que tem necessidade que diferentes eixos operativos atuem ativamente e em sinergia. Uma atuação dos eixos políticos, sociais, saúde, multidisciplinar e intergeracional oferece oportunidade de participação ativa da pessoa idosa, permitindo um envolvimento social, autocuidado da sua saúde, autoestima e qualidade de vida.

Entre esses eixos operativos, a educação física tem a sua posição bem assente. Os benefícios da prática de exercício físico regular na saúde e no bem-estar da pessoa idosa estão bem sustentados, visto a divulgação da esmagadora literatura científica hoje disponível para o leitor. ${ }^{3,24,26}$ Por outro lado, ainda parece ser necessário trabalhar a concepção do termo "envelhecer" na sociedade brasileira. Existe um objeto cultural de uma noção de associação de envelhecimento e velhice com doença, perda, incapacidade e dependência que contribui vivamente para um estereótipo negativo e preconceituoso. O impacto desse entendimento não é só negativo no âmbito social, mas extensivo à autoconcepção da pessoa idosa e sua motivação para integrar-se e participar. ${ }^{42}$

Com base nesse contexto, pode ser dito em primeiro lugar que a educação física, hoje, quando atua com a população idosa, assume um posicionamento construtivo e positivo. Entende-se que, na diversidade de tipos de práticas de exercício físico adotados para essa população, que os objetivos se situam em potencializar as capacidades físicas e cognitivas as quais deverão ser estimuladas e mantidas. Nesta linha de atuação se contraria propostas que se esgotam em ajustarem-se, adaptarem-se às capacidades diminuídas pelo processo de senescência. ${ }^{14} \mathrm{~A}$ educação física tem um importante papel social em educar e preparar a sociedade para envelhecer.

Um outro ponto importante referente às perspectivas de atuação da educação física, é que esta tem a função de levar as pessoas a conhecerem a forma que envelhecem. Entende-se que a forma que se envelhece causa influência direta no processo de envelhecimento. ${ }^{43}$ Essas formas, que variam desde um envelhecimento bem-sucedido a um dito patológico, diante de medidas de mudança de conduta para estilo de vida saudável, são mostradas como atitudes proativas com importante proteção na saúde e qualidade de vida. ${ }^{44}$

Descrito isso, quando se trata de programas de atividade física regular para a pessoa idosa, eles têm importante necessidade em disporem-se de variabilidade nas propostas práticas e na oportunidade de acesso. Essa ideia é sustentada pela Pesquisa Nacional de Saúde ${ }^{10}$, que evidencia a necessidade de os programas públicos levarem mais informação, serem capazes em criar ambientes favoráveis, assim como gerarem oportunidade de participação e inclusão dessa população, sem diferenciação etária e demais características. ${ }^{10}$ Unicamente deverão estar adequados às características e necessidades dessas pessoas. Os programas de atividade física regular para idosos têm em sua grande maioria a participação de idosos jovens (60-79 anos). .310 Uma visão mais diversificada das condições de atuação é ponto a ser considerado.

Quanto à relevância da participação da pessoa idosa de forma ativa para sua saúde, temos como exemplo a estratégia do programa "Tomando Controle da Sua Saúde”, promovido pela Organização Pan-Americana de Saúde, uma proposta que integra a educação e um conjunto multidisciplinar de práticas em que o indivíduo aprende a cuidar e ser responsável pela sua própria saúde. ${ }^{44}$ No trato com a pessoa idosa interessa que ela aprenda e compreenda que exercício físico ou esporte não correspondem unicamente a uma atividade esportiva ou de lazer a ser integrada na sua rotina, e 
sim uma medida preventiva de manutenção da saúde com baixo custo.

A educação física na vida da pessoa idosa assume um papel de prevenção e promoção da sua saúde, porém, para ampliar suas potencialidades, depende de empoderamento, comunitário, político, social, da saúde e da comunidade científica. A colocação do profissional de educação física nas unidades de saúde, ${ }^{11}$ instituições de longa permanência e na atenção à comunidade é ponto emergente a ser valorizado na busca do avanço da promoção da saúde dessa população.

\section{Novas perspectivas}

A OMS, através da Carta de Toronto, faz um apelo aos países, sobretudo aos países em desenvolvimento, para que estabeleçam Planos de Ação para Atividade Física. ${ }^{45}$ Nesse sentido, o executivo da OMS, em parceria com alguns países europeus, se empenha para a elaboração de um projeto-modelo de plano de ação global para a revitalização da atividade física a ser aprovado em 2018.45

Conhecedores e conscientes das consequências do sedentarismo na saúde, condições de vida e qualidade de vida e economia em saúde pública, entendemos que a contribuição da educação física no processo de envelhecer é agora e para as próximas décadas uma profissão de extrema importância na conservação dos anos vividos com saúde, i.e., anos de vida ativo.

A Carta de Toronto advoga pela implantação de oportunidades sustentáveis de programas de atividade física e estilo de vida saudável. Nesse sentido, uma das recomendações da carta é que sejam seguidos e adaptados à cultura e às condições socioeconômicas os modelos que já tenham demonstrado serem efetivos. Como descrito anteriormente, há necessidade de alargar oportunidades e de evidenciar resultados estatisticamente econômicos (custo-utilidade/custo efetividade) pela aplicação de programas.

As oportunidades de acesso deverão ser consideradas quanto à localização, devendo ocorrer de forma heterogênea (aberto para todos), adequação do programa às características e necessidades dos idosos e ocorrer em maior número por programas comunitários em que não exista um início e um fim, típicos do programa em ambiente acadêmico. $\mathrm{O}$ ambiente acadêmico deverá procurar implementar programas com continuidade e diversificados quanto às faixas etárias e condições funcionais. A gratuidade nesses programas é ponto decisivo à adesão e permanência. Assim sendo, a valorização da contratação dos profissionais de educação física e do apoio financeiro dos outros eixos operativos, como educação, político e social, é um aspecto a ser fortemente considerado. ${ }^{11,44}$
Quanto aos resultados econômicos mostrados por esses modelos de programas de atividade física regular para a pessoa idosa, eles serão conhecidos e compreendidos como forma de contenção de custos em saúde, visto o baixo custo em realizar exercício físico e suas consequências benéficas na saúde física e mental. ${ }^{37}$

Deduzimos, a partir do exposto, que existem novas perspectivas a serem analisadas, consideradas e valorizadas num ritmo temporal curto. Uma maior transversalidade entre eixos operativos no Brasil é necessária para a ampliação dos programas de atividade física regular para o idoso. Como referenciado por diversos estudos, os profissionais de educação física especializados e conhecedores do exercício da orientação, avaliação e prescrição têm plenas condições de atuação nessa população. É de extrema importância que o profissional de educação física que atue no campo da gerontologia preventiva seja conhecedor dos aspectos fisiológicos, psicológicos e socioafetivos relacionados com o envelhecimento e das mudanças ocorrentes no comportamento motor ao longo da vida. Por fim, que tenha o domínio dos princípios, objetivos e das metodologias subjacentes ao desenvolvimento e controle de programas e prescrição de exercício para pessoas idosas.

\section{CONCLUSÕES}

As contribuições da educação física para a pessoa idosa estão bem definidas e são entendidas pelas comunidades médica e científica como componente indispensável para a conservação da saúde e anos vividos com saúde. As novas perspectivas se estabelecem no empoderamento ativo dos eixos operativos na política de envelhecimento ativo. As propostas de programas de atividade física regular para a pessoa idosa carecem de alargar oportunidades e evidenciar resultados estatisticamente econômicos (custo-utilidade/custo efetividade) pela aplicação de programas.

\section{REFERÊNCIAS}

1. Organización Mundial de la Salud. Informe mundial sobre el envejecimiento y la saludd [Internet]. Geneva: OMS; 2015 [capturado em 19 nov. 2018]. Disponível em: https://apps.who.int/iris/bitstream/handle/10665/186471/ WHO FWC ALC 15.01 spa.pdf; jsessionid=7A5C7D79 6E600B $1 \mathrm{E} 689206 \mathrm{C} 3840 \mathrm{AA} 753$ ? sequence $=1$. https://oi. org/10.30875/17eb8f18-es

2. Instituto Brasileiro de Geografia e Estatística. Diretoria de Pesquisas - DPE, Coordenação de População e Indicadores Sociais [Internet]. Rio de Janeiro: IBGE; 2017 [capturado em 19 nov. 2018]. Disponível em: https://ww2. ibge.gov.br/home/disseminacao/eventos/missao/principais enderecos.shtm. https://doi.org/10.1007/bf00461158 
3. Squarcini CFR, Rocha SV, Munaro HLR, Benedetti TRB, Almeida FA. Physical activity programs for elderly persons: an evaluation of Brazilian scientific production using the RE-AIM framework. Rev Bras Geriatr Gerontol. 2015;18(4):909-20. https://doi.org/10.1590/1809. 9823.2015 .14103

4. World Health Organization. Active ageing: a policy framework. Geneva: WHO; 2002.

5. Camargos MCS, Gonzaga MR. Viver mais e melhor? Estimativas de expectativa de vida saudável para a população brasileira. Cad Saúde Pública. 2015;31 (7):1460-72. https://doi.org/10.1590/0102$311 \times 00128914$

6. Aires A, Pelisoli M. Biomechanical analysis of gait in active older adults. Proceedings of $22 \mathrm{nd}$ Annual Congress of the European College of Sport Science. 2017.

7. Loureiro A, Aires AG, Printes CB, Marquesan F, Faggiani F, Tarouco I, Castro L, Brandt L, Pelisoli M, Baptista R, Sartori R, Gomes S, Irigaray T, Vilar T. Análise da capacidade funcional de idosos através do teste de $6 \mathrm{~min}$ walking com o sensor inercial. Anais do XVII Congresso Brasileiro de Biomecânica. Porto Alegre: Pontifícia Universidade Católica do Rio Grande do Sul; 2017. https://doi.org/10.5327/z2447-211520171700051

8. Tomporowski PD. The effects of physical and mental training on the mental abilities of older adults. J Aging Phys Act. 1997;5(1):9-26.

9. McAuley E, Elavsky S. Self-efficacy, physical activity, and cognitive function. In: Spirduso WW, Poon LW, Chodzko-Zajko W, editors. Aging, exercise, and cognition series: exercise and its mediating effects on cognition. Champaign: Human Kinetics; 2008. p. 69-84.

10. Biehl-Printes C, Brauner FO, Rocha JP, Oliveira G, Neris J, Rauber B, Bós AJ. Prática de exercício físico ou esporte dos idosos jovens e longevos e o conhecimento dos mesmos em programas públicos: pesquisa nacional de saúde 2013. Pajar. 2016;4(2):47-53. https://doi. org/10.15448/2357-9641.2016.2.25276

11. Ferreira AB, Minanni CA, Paganini CBL, La Falce TC, Todeschini AB, Eras AE, Chekin G, Motta BR, Carneiro Junior N. Programa de atenção particularizada ao idoso em unidades básicas de saúde. Saúde Soc. 2009;18(4):776-86. https://doi.org/10.1590/s0104-12902009000400020

12. Sá PHVO, Cury GC, Ribeiro LCC. Atividade física de idosos e a promoção da saúde nas unidades básicas. Trab Educ Saúde. 2016;14(2):545-58. https://doi. org/10.1590/1981-7746-sip00117

13. Nascimento CMC, Ayan C, Cancela JM, PereiraI JR, Andrade LP, GaruffiI M, Gobbi S, Stella F. Exercícios físicos generalizados capacidade funcional e sintomas depressivos em idosos brasileiros. Rev Bras Cineantropom Desempenho Hum. 2013;15(4):486-97. https://doi. org/10.5007/1980-0037.2013v15n4p486

14. Printes C, Costa A, Marmeleira J, Carús P. Gerontomotricidade: programa de exercício físico para pessoas idosas. Lisboa: Self Desenvolvimento Pessoal; 2016.
15. Caspersen CJ, Powell KE, Christenson GM. Physical activity, exercise, and physical fitness: definitions and distinctions for health-related research. Public Health Rep. 1985;100(2):126-31.

16. Chodzko-Zajko WJ. Psychological and sociocultural aspects of physical activity for older adults. In: Jones CJ, Rose DJ. Physical activity instruction of older adults. Champaign: Human Kinetics; 2005. chap. 3.

17. Siqueira FV, Nahas MV, Facchini LA, Silveira DS, Piccini RX, Tomasi E, Thumé E, Hallal PC. Aconselhamento para a prática de atividade física como estratégia de educação à saúde. Cad Saúde Pública. 2009;25(1):203-13. https://doi. org/10.1590/s0102-311x2009000100022

18. Rocha SV, Almeida MMG, Araújo TM, Santos LB, Rodrigues WKM. Fatores associados à atividade física insuficiente no lazer entre idosos. Rev Bras Med Esporte. 2013;19(3):191-5. https://doi.org/10.1590/s151786922013000300009

19. Gonzaga JM, Barros SEB, Lisboa MGC, Barbieri FA, Gobb LTB. Efeitos de diferentes tipos de exercício nos parâmetros do andar de idosas. Rev Bras Med Esporte. 2011;17(3):66-170. https://doi.org/10.1590/s151786922011000300003

20. Salin MS, Mazo GZ, Cardoso AS, Garcia GS. Atividade física para idosos: diretrizes para implantação de programas e ações. Rev Bras Geriatr Gerontol. 2011;14(2):197-208. https://doi.org/10.1590/s1809. 98232011000200002

21. Rosário CG, Ribeiro MM, Silva VAP. Práticas corporais desenvolvidas com idosas participantes do projeto Educar Para a Atividade Física (EPAF) da Universidade do Estado da Bahia e variáveis da aptidão física. In: Campos HJC, Pitanga FJG, organizadores. Práticas investigativas em atividade física e saúde. Salvador: EDUFBA; 2013. p. 191-204. https://doi.org/10.7476/9788523212209

22. Castro PC, Tahara N, Rebelatto JR, Driusso P, Aveiro MC, Oishi J . Influência da universidade aberta da terceira idade (UATI) e do programa de revitalização (REVT) sobre a qualidade de vida de adultos de meia-idade e idosos. Rev Bras Fisioter. 2007;11(6):461-7. https://doi. org/10.1590/s1413-35552007000600007

23.Antikainen I, Ellis R. A RE-AIM evaluation of theory-based physical activity intervetions. J Sport Exerc Psychol. 2011;33(2):198-214. https://doi.org/10.1123/ jsep.33.2.198

24. Teixeira CS, Pereira EF, Rossi AG. A hidroginástica como meio para manutenção da qualidade de vida e saúde do idoso. Acta Fisiátrica. 2007;14(4):226-32.

25. Silva AG, Ribeiro JC. Hidroginástica na terceira idade. Ágora. 2010;17(2):49-59.

26. Coelho BS, Souza LK, Bortoluzzi R, Roncada C, Tiggemann CL, Dias CP. Comparação da força e capacidade funcional entre idosos praticantes de musculação, hidroginástica e não praticantes de exercícios físicos. Rev Bras Geriatr Gerontol. 2014; 17 (3):497-504. https://doi.org/10.1590/1809-9823. 2014.13046 
27. Ueno DT, Gobbi S, Teixeira CVL, Sebastião E, Prado AKG, Costa JLR, Gobbi LTB. Efeitos de três modalidades de atividade física na capacidade funcional de idosos. Rev Bras Educ Fís Esporte. 2012;26(2):273-81. https://doi. org/10.1590/s1807-55092012000200010

28. Almeida MAB, Pavan B. Os benefícios da musculação para a vida social e para o aumento da autoestima na terceira idade. Rev Bras Qualidade Vida. 2010;2(2):9-17. https://doi.org/10.3895/s2175-08582010000200002

29. Silva WA. Atividade física e terceira idade: um estudo de caso sobre as atividades oferecidas pelo grupo viver feliz, do município de Águas Lindas de Goiás [Monografia]. Brasília: UnB; 2012.

30. Ribeiro CSP, Bolzan G, Zappe AR, Gasparetto A, Mai C. A artrose na terceira idade: um estudo de caso [Internet]. Santa Maria; 2011 [capturado em 19 nov. 2018]. Disponível em: https://pt.slideshare.net/AdrianoPires/ artrose-na-terceira-idade. https://doi.org/10.22478/ ufpb.1809-4775.2018v14n1.40031

31. Benedetti TRB, Schwingel A, Gomez LSR, ChodzkoZajko W. Programa "VAMOS" (Vida Ativa Melhorando a Saúde): da concepção aos primeiros resultados. Rev Bras Cineantropom Desempenho Hum. 2012;14(6):723-37. https://doi.org/10.5007/1980-0037.2012v14n6p723

32. Bonganha V, Modeneze DM, Madruga VA, Vilarta R. Effects of resistance training (RT) on body composition, muscle strength and quality of life $(\mathrm{QoL})$ in post menopausal life. Arch Gerontol Geriatr. 2012;54(2): 361-5. https://doi.org/10.1016/j.archger.2011.04.006

33. Hernandez SSS, Coelho FGM, Gobbi S, Stella F. Efeitos de um programa de atividade física nas funções cognitivas, equilíbrio e risco de quedas em idosos com demência de Alzheimer. Rev Bras Fisioter. 2010;14(1):68-74. https://doi. org/10.1590/s1413-35552010000100011

34. Terra NL, Printes CB, Terra PA, Oppermann R. Doenças geriátricas \& exercícios físicos. Porto Alegre: EDIPUCRS; 2016

35. Tully MA, Cupples ME, Chan WS, McGlade K, Young IS. Brisk walking, fitness, and cardiovascular risk: a randomized controlled trial in primary care. Prev Med. 2005;41(2):622-8. https://doi.org/10.1016/j. ypmed.2004.11.030

36. American College of Sports Medicine. Exercício para pacientes com doença arterial coronariana. Rev Bras Med Esporte. 1998;4(4):122-6. https://doi.org/10.1590/s1517. 86921998000400006

37. Gusi N, Reyes MC, Gonzalez-Guerrero JL, Herrera E, Garcia JM. Cost-utilityof a walking programme for moderately depressed, obese, or overweight elderly women in primary care: a randomized controlled trial. BMC Public Health. 2008;8:231. https://doi.org/10.1186/ 1471-2458-8-231

38. Oliveira AC, Oliveira NMD, Arantes PMM, Alencar MA. Qualidade de vida em idosos que praticam atividade física: uma revisão sistemática. Rev Bras Geriatr Gerontol. 2010;13(2):301-12. https://doi.org/10.1590/s1809. 98232010000200014
39. Lustosa LP, Máximo PLS, Coelho FM, Pereira DS, Silva JP, Parentoni AN, Dias RC, Dias JMD. Impact of an exercise program on muscular and functional performance and plasma levels. Arch Phys Med Rehabil. 2013;94(4):660-6. https://doi.org/10.1016/j.apmr.2012.11.013

40. Burke TN, França FJ, Meneses SRF, Cardoso VI, Marques AP. Postural control in elderly persons with osteoporosis: efficacy of an intervention program to improve balance and muscle strength: a randomized controlled trial. Am J Phys Med Rehabil. 2010;89(7):549-56. https://doi. org/10.1097/phm.0b013e3181ddccd2

41. Parlamento Europeu. 2012: Ano europeu do envelhecimento activo e da solidariedade entre gerações [Internet]. Lisboa; 2012 [capturado em 19 nov. 2018]. Disponível em: http://www.europarl.europa.eu/news/pt/ headlines/eu-affairs/20120106STO34946/2012-ano-europeudo-envelhecimento-activo-e-da-solidariedade-entregeracoes. https://doi.org/10.18055/finis1470

42. Printes CB. A busca da qualidade de vida e a contribuição da Universidade aberta para terceira idade. Rev SORBI. 2013;1(1):19-21. https://doi.org/10.18308/2318-9983. $2013 v \ln 1.12$

43. Moraes EN. Princípios básicos de geriatria e gerontologia. Brasília: Coopmed; 2008.

44. Organización Panamericana de la Salud. Promoción de la salud: una antología. Rev Esp Salud Pública. 1997;71(3):317-20. https://doi.org/10.1590/s113557271997000300010

45. The bangkok declaration on physical activity for global health and sustainable development. Proceedings of the 6th ISPA International Congress on Physical Activity and Public Health. Thailand; 2016.

\section{AUTHORS:}

CLARISSA BIEHL-PRINTES

Docente Convidada do Instituto de Geriatria e Gerontologia da Pontifícia Universidade Católica do Rio Grande do Sul (PUCRS), Porto Alegre, RS, Brasil. E-mail: clarissaprintes@hotmail.com

Orcid: http://orcid.org/0000-0002-1464-3731

FABIANE DE OlIVEIRA BRAUNER

Pesquisadora, Pontifícia Universidade Católica do Rio Grande do Sul (PUCRS), Porto Alegre, RS, Brasil.

E-mail: braunerpro@gmail.com

Orcid: http://orcid.org/0000-0001-5763-7537

NEWTON LUIZ TERRA

Diretor do Instituto de Geriatria e Gerontologia da Pontifícia Universidade Católica do Rio Grande do Sul (PUCRS), Porto Alegre, RS, Brasil.

E-mail: terranl@pucrs.br

Orcid: http://orcid.org/0000-0002-7904-3649 